\title{
EDITORIAL
}

\section{Will Molnupiravir be the First Oral Treatment for High-Risk Non- Hospitalized Patients with Mild-Moderate COVID-19?}

The first case of SARS-CoV-2 infection in United States was confirmed on January 19, 2020, in a man who had returned home to Snohomish County, Washington on January 15, 2020, after traveling to Wuhan, China. Since the outbreak of this pandemic, significant progress has been made in the development of vaccines that are used in preventing SARS-CoV-2 infection. To date, over 392 million doses of the three vaccines approved under the Emergency Use Authorization (EUA) have been administered in the United States (1). Given the safety and efficacy of Pfizer/BioNTech mRNAbased vaccine and after rigorous scientific review of available data, U.S. Food and Drug Administration (FDA) has fully approved this vaccine which is now marketed under the brand name Comirnaty for use in people 16 years of age or older (2).

With well over 21 months into this pandemic, we still do not have any treatment that has received full FDA approval for COVID-19. Numerous agents have been approved under EUA for the treatment of SARS-CoV-2 infection in high-risk patients and several clinical trials are currently underway to discover the elusive drug for this purpose. The only agents currently approved for outpatient use under EUA for the treatment of mild-moderate SARSCoV-2 infection are the three monoclonal antibody cocktails that require intravenous infusion thus limiting their utility on a widespread basis (3).

An ideal drug for the treatment of COVID-19 would be an oral medication that could be administered out-of-hospitals to patients with mild-moderate disease. The full genome of SARSCoV-2 has been identified and researchers have also used advanced microscopy techniques to map the 3D structure of the virus proteins in detail (4). Together, this information has propelled the search for new drugs to treat SARS-CoV-2 infection that specifically target its structure and functions. In a recent non-peer reviewed article, Fischer, et.al., have claimed that Molnupiravir - an experimental anti-viral agent when administered orally in patients with mild to moderated SARS-CoV-2 infection reduced the risk of hospitalization or death by about $50 \%$ as compared to the placebo (5). These observations prompted a recent news release by Merck (who developed Molnupiravir in partnership with Ridgeback Biotherapeutics) indicating that they will be submitting an investigational new drug application to the FDA seeking EUA for oral treatment of patients with mild-moderate COVID$19(6)$.

Molnupiravir was originally developed by Drug Innovations at Emory University to treat influenza (7). It is a pro-drug that is metabolized into a ribonucleoside (RNA) analog that resembles cytidine - a critical component of RNA. Molnupiravir's anti-viral activity is exerted by a process known as lethal mutagenesis or viral error catastrophe. Essentially, during replication, the viral RNA incorporates Molnupiravir which leads to downstream mutations resulting in catastrophic errors that adversely impact the survival of the virus.

The non-peer reviewed unpublished interim data that Merck is planning to use to obtain an EUA emanates from interim analysis of a Phase 3 trial (MOVe-OUT) which was a randomized, controlled, double-blinded assessment of Molnupiravir versus placebo in non-hospitalized adults with mild to moderate COVID-19 with symptom onset within 5 days prior to their randomization. It is important to note that the eligible trial participants had $\geq 1$ risk factor (such as obesity, diabetes, old age, etc.) that are associated with poor COVID-19 outcomes. The primary outcome of this trial was to determine the efficacy of Molnupiravir by evaluating the percentage of participants who are hospitalized and/or who have died through 29 days postrandomization. 
The key observations of an interim analysis of this Phase 3 (MOVe-OUT) study were:

- Molnupiravir reduced the patient's risk of COVID-19 hospitalization or death by approximately $50 \%$ :

- Only 28 (7.3\%) patients who received Molnupiravir were hospitalized, versus 53 (14.1\%) patients who were administered placebo

- As compared to eight (8) patients in the placebo arm, no patients who received Molnupiravir died through 29 days post-randomization

- Molnupiravir was consistently effective against Delta, Gamma, and Mu SARS-CoV2 variants

- Adverse events occurred in $35 \%$ and $40 \%$ of patients treated with Molnupiravir versus placebo, respectively, however, trial discontinuation due to adverse event was lower $(1.3 \%)$ in the treatment group as compared to the placebo group $(3.4 \%)$

With the human death toll in the US due to COVID19 now outstripping that during the 1918 Influenza pandemic, the quest to discover an oral drug that is approved by the FDA to treat patients with mildmoderate SARS-CoV-2 infection has achieved the revered status of the Holy Grail in the field of medicine and public health. Interim data analysis suggests that Molnupiravir has the potential to be a "drug of interest" as an oral agent to treat patients with mild-moderate COVID-19. While this is indeed very encouraging, submission/review of data by the FDA and other regulatory agencies along with publication of long-term follow-up of ideally a larger cohort of patients in peer-reviewed journal is quintessential to unequivocally establish the efficacy of Molnupiravir in the oral treatment of non-hospitalized high-risk patients with mildmoderate SARS-CoV-2 infection.

\section{References}

1. COVID-19 dashboard. John Hopkins University of Medicine. 2021. https://coronavirus.jhu.edu/map.html (Accessed October 02, 2021; 21:48 p.m. CST)

2. FDA approves first COVID-19 vaccine. 2021 https://www.fda.gov/news-events/press-announcements/fdaapproves-first-covid-19-vaccine
3. Available COVID-19 treatment option. 2021. https://combatcovid.hhs.gov/i-have-covid-19-now/availablecovid-19-treatment-options

4. Nazario-Toole AE, Xia H, Gibbons TF. Whole-genome Sequencing of SARS-CoV-2: Using Phylogeny and Structural Modeling to Contextualize Local Viral Evolution [published online ahead of print, 2021 Feb 20]. Mil Med. 2021; usab031. doi:10.1093/milmed/usab031

5. Fischer W, Eron JJ, Holman W, Cohen MS, Fang L, Szewczyk LJ, Sheahan TP, Baric R, Mollan KR, Wolfe CR, Duke ER, Azizad MM, Borroto-Esoda K, Wohl DA, Loftis AJ, Alabanza P, Lipansky F, Painter WP. Molnupiravir, an Oral Antiviral Treatment for COVID-19. medRxiv [Preprint]. 2021 Jun 17:2021.06.17.21258639. doi: 10.1101/2021.06.17.21258639. PMID: 34159342; PMCID: PMC8219109.

6. Merck and Ridgeback's Investigational Oral Antiviral Molnupiravir Reduced the Risk of Hospitalization or Death by Approximately 50 Percent Compared to Placebo for Patients with Mild or Moderate COVID-19 in Positive Interim Analysis of Phase 3 Study. Oct 01, 2021. https://www.merck.com/news/merck-and-ridgebacksinvestigational-oral-antiviral-molnupiravir-reduced-the-risk-ofhospitalization-or-death-by-approximately-50-percent-

compared-to-placebo-for-patients-with-mild-or-moderat/

7. Toots M, Yoon JJ, Cox RM, Hart M, Sticher ZM, Makhsous N, Plesker R, Barrena AH, Reddy PG, Mitchell DG, Shean RC, Bluemling GR, Kolykhalov AA, Greninger AL, Natchus MG, Painter GR, Plemper RK. Characterization of orally efficacious influenza drug with high resistance barrier in ferrets and human airway epithelia. Sci Transl Med. 2019 Oct 23;11(515): eaax5866. doi: 10.1126/scitranslmed. aax5866. PMID: 31645453; PMCID: PMC6848974

\section{Sohail Rao, MD, MA, DPhil}

Executive Vice President, DHR Health, 5501 S. McColl Road, Edinburg, Texas

President \& Chief Executive Officer, DHR Health Institute for Research \& Development, 5323 S. McColl Road, Edinburg, Texas

\section{Corresponding author email: s.rao@dhr-rgv.com}

Disclosures: None

ORCID: Sohail Rao: https://orcid.org/0000-0001-50279992

\section{Manish Singh, MD, FACS}

Chief Executive Officer, DHR Health, 5501 S. McColl Road, Edinburg, Texas

Disclosures: None

ORCID: Manish Singh: https://orcid.org/0000-00034146-3282 